\title{
A MODIFICATION. OF THE LEPROMIN TEST
}

by J. A. Kinnear Brown, B.SC., M.D., M.R.C.S., D.T.M.

Specialist Leprologist, Uganda

Lepromin is an antigen prepared by grinding $1 \mathrm{gm}$. of autoclaved bacteriologically positive tissue in $20 \mathrm{ml}$. of normal saline, a concentration of $1: 20$. The test is made by injecting $0.1 \mathrm{ml}$. intradermally. At the end of 24 to 48 hours, there is a tuberculin like response which rapidly fades, to be succeeded in most instances by a nodular infiltration which reaches its maximum size in three to four weeks. The early reaction is allergic, less constant, and may include a non-specific element due to tissue debris; the late result, the Mitsuda reaction, is thought to depend on the presence of whole bacilli and to indicate resistance to infection, because those with a negative Mitsuda develop the more serious forms of leprosy, and those with a strongly positive reaction, the milder forms. The late reaction is measured as follows :-

Infiltrations of 1 and $2 \mathrm{~mm}$. are designated \pm doubtf ul

Infiltrations of 3 and $4 \mathrm{~mm}$. are designated + weakly positive

Infiltrations of $5 \mathrm{~mm}$. and over are designated ++ positive

Ulceration is designated +++ strongly positive.

It is this late reaction which is referred to as the lepromin test in this report.

In the absence of a culture, the only satisfactory source of lepromin is the tissue of lepromatous patients. The supply of antigen is limited by their number and may not be equal to the demand. Attempts have therefore been made particularly by Floch, Diniz and Neto, and Schujman to discover the extent to which lepromin can be diluted and give results which can be correlated with those produced by the standard antigen.

In the majority of adults, the results of the lepromin and tuberculin tests coincide and it would simplify matters if tuberculin could be used to detect those who respond weakly to lepromin. The parallelism is not sufficiently close, however, and the measure of agreement depends to some extent on the test dose of tuberculin. In any case, the two tests are fundamentally different. The response to PPD reflects a definite experience with the tubercle bacillus but says little about the future; that to lepromin says little about the past (positive reactions occur quite commonly outside endemic areas) but more about the future, because it indicates reasonably well what will happen if the individual encounters infection. Until a successf ul culture is made or a new test evolved, experiments must continue until the present one has been so modified that its use on a large scale becomes practicable. 
Preliminary Work. 269 patients in the Buluba Leprosarium were lepromin tested by the ordinary intradermal method using dilutions of $1: 20,1: 300$ and $1: 750$. The percentage that gave positive reactions dropped from $86 \%$ with $1: 20$, to $53 \%$ with $1: 300$ and to $37 \%$ with $1: 750$. The percentage that gave doubtful reactions increased from $12 \%$ with $1: 20$ to $23 \%$ with $1: 300$ and persisted at this level at $1: 750$. The percentage of negative results increased from $2 \%$ with $1: 20$ to $23 \%$ with $1: 300$ and $41 \%$ with $1: 750$. In some of those who remained positive throughout there was a reduction in the size of the infiltration with the more dilute antigens but this was less noticeable in those who were initially strongly positive. In those who were tested with $1: 100$, there was little difference from the result with normal antigen.

\section{A Multipuncture Lepromin Test}

It was decided to investigate the possibility of using a multipuncture apparatus to carry out a modified lepromin test, using normal lepromin and a dilution of $1: 100$. The Heaf apparatus provides a convenient method of tuberculin testing, particularly where children are involved. It produces six tiny punctures arranged in a circle and these are made through antigen smeared on the skin. The reaction which follows is read after 72 hours.

A positive reading is indicated by a palpable induration of at least four puncture points, and is graded as follows:-

Grade 1. Discrete palpable induration at the puncture points.

Grade 2. A coalescing of the indurated points to form an oedematous ring.

Grade 3. More extensive induration forming. a coin pattern approximately $10 \mathrm{~mm}$. in diameter.

Grade 4. More extensive induration with possible sloughing of the centre.

The lepromin used was made up to strengths of 1:20 and 1:100 to which $10 \%$ glycerine was added (as in PPD). A small quantity was placed on the flexor surface of the forearm. The plate of the multipuncture apparatus, sterilised by flaming was pressed evenly on the moistened area and the plunger driven home, when the six needles penetrated the plate and made the punctures. The tests with the weaker antigen were made at a higher level on the arm and the patients were made to sit after testing with their forearms supported horizontally until the antigen had dried. Where an ordinary intradermal test was done at the same time, it was placed at a lower level, so that there was no chance of more concentrated antigen running 
down by gravity and contaminating a test made with a weaker dilution.

One hundred and ninety-two patients at the Kumi-Ongino Leprosarium were chosen of all ages and at various stages of treatment, none of whom was clinically lepromatous, and none of whom had been previously tested. 27 were tested with standard lepromin in the ordinary way (referred to for simplicity as 'the intradermal method'). These and the 165 others were tested at the same time percutaneously with the Heaf apparatus with lepromin in the two strengths.

In all the multipuncture tests, there was an immediate traumatic response which rapidly faded. This was followed by another similar in appearance which was differentiated as visible, palpable or prominent. At the apices of some of the latter, a pinpoint vesicle and crust eventually formed. In contrast with PPD, the swelling at each puncture remained discrete, there was fusion only in a very small minority and in no case did the whole area become involved. There was no distinctive early and late reaction. The response appeared at a different time in each individual but once it appeared, it persisted and was usually progressive. Of the 192 patients, the numbers with a palpable reaction at various time intervals were:-

$\begin{array}{cr}\text { Time interval } & \text { Number rear } \\ 24-48 \text { hours } & 24 \\ 72 \text { hours } & 53 \\ 5 \text { days } & 106 \\ 1 \text { week } & 115 \\ 2 \text { weeks } & 149 \\ 3 \text { weeks } & 146 \\ 4 \text { weeks } & 136\end{array}$

In some of the others, and in many of those who had a palpable reaction, there remained eventually a pinhead circle of hyperpigmentation at the site of each puncture. This was discounted. The chief difficulty at first was in deciding whether a response was palpable, this having been chosen as the arbitrary standard of positivity. This difficulty lessened with experience.

All the 27 patients tested intradermally had clinical tuberculoid leprosy and were Mitsuda positive, with a late infiltration of $5 \mathrm{~mm}$. or more. Only 12 of these produced a ring of papules with the multipuncture tests; the other 15 showed either no visible change or none that was palpable. This work was repeated at the Buluba Leprosarium on 23 patients who had been Mitsuda positive one to three years previously, and who had been tested with the multipuncture apparatus three months earlier, with positive results. They were retested 
simultaneously by both methods on the same arms. Of the Mitsuda results 9 were now stronger, 10 were the same, and 4 were weaker; by the percutaneous method, none were stronger, 7 were the same and 16 weaker. The positive Mitsuda response appeared, therefore, to depress that to the percutaneous method in some patients if done at the same time, thus nullifying this method of calibrating one test in terms of the other.

In the patients who showed a palpable reaction to the percutaneous method, that to the 1:100 dilution was of ten stronger. This was an anomaly difficult to explain. All the lepromin was from the same batch. All the tests with the normal antigen were done first before any dilution was made. A different and previously unused apparatus was used for the two series.

Subsequently, this was repeated on a small group at the Buluba Leprosarium with glycerinated lepromin, when 6 out of 11 patients showed a stronger reaction to $1: 100$, but weaker to $1: 300$, or $1: 750$. When unglycerinated lepromin was used, and the dilution $1 ; 100$ made with normal saline, there was little difference, except that the normal lepromin gave a slightly stronger reaction.

At the Buluba and Nyenga Leprosaria, 216 patients whose lepromin status had been determined intradermally one to three years earlier were now examined by the percutaneous method, using lepromin 1:20 without glycerine. The agreement between the original and the new tests was very close. If anything, there were slightly more positives by the later tests among the tuberculoid patients but this was what one would expect as the previous dose of antigen would have had a stimulating effect. Where there was a change in the opposite direction, it was among the indeterminate and dimorphous patients who are always the more unstable.

\section{Correlation of the Two Methods of Lepromin Testing}

When the intradermal and multipuncture tests were done on the arm side by side, the multipuncture test was frequently suppressed, making it difficult to establish any correlation. Twenty-one lepromin positive patients were now tested simultaneously on the forearm and back, using 1:20 lepromin intradermally. All gave a late positive response of more than $5 \mathrm{~mm}$. The reactions in each individual were not always the same but any difference was small and it was not in favour of either site. The site of injection did not, therefore, affect the response. If there was any suppression of one test by the other it was not considerable.

Twenty adults were then tested simultaneously by the intradermal method, using 1:20 lepromin on the arm and 1:100 lepromin on the back. All gave a late positive response of $4 \mathrm{~mm}$. or more.

The addition of $25 \%$ to the size of the infiltration caused by the 
weaker antigen gave the grade of positivity produced by the stronger. It did not always give the exact measurement, but it did give the grading.

The addition of $25 \%$ to smaller infiltrations of 1,2 and $3 \mathrm{~mm}$. (ignoring fractions of a millimetre) would not alter their grade. With this method of correlation, an infiltration of $4 \mathrm{~mm}$. is the only one that would be put into a higher grade, and this is what happenedan infiltration of $4 \mathrm{~mm}$. to 1:100 lepromin was accompanied by a $5 \mathrm{~mm}$. response to the $1: 20$ antigen.

In another series, in which the reaction to intradermal 1:20 lepromin had been determined three months earlier, the response to $1: 100$ lepromin had a similar relationship. Adding $25 \%$ did not put any patient into the wrong grade. As a practical measure, therefore, where it is desired to know more than whether the reaction is positive or negative 1:100 lepromin can be substituted.

Alternatively the same result in degrees of positivity would be achieved by adjusting the Standards for $1: 100$ lepromin as follows :-

Infiltrations of 1 and $2 \mathrm{~mm}$. \pm doubtful.

Infiltrations of $3 \mathrm{~mm}$. + weakly positive.

Infiltrations of $4 \mathrm{~mm}$. or more ++ positive

Ulceration +++ strongly positive.

If there is ulceration, it usually occurs with both antigens but it is less severe with the $1: 100$ dilution.

Forty children were now lepromin tested by the intradermal method in the interscapular area, and by the multipuncture methor on the forearm using 1:20 lepromin in isotonic saline in both instances. Between the third and fourth week, 37 were positive to both tests and the responses in each patient were proportional. In the earlier work the suppression of the multipuncture test by the intradermal must have been due more to local than central action.

Three failed to give a reaction to the multipuncture method nor did they do so when it was repeated while the ordinary Mitsuda response was still present. This could only mean that suppression is not entirely a local phenomenon.

The following arbitrary grading was now adopted:

Grade I Four or more palpable and discrete papules

Grade II Four or more prominent and discrete papules

Frade III Four or more prominent papules with pinpoint ulceration at one of them, or all tending to coalesce.

Table I compares the results obtained with both tests. 
TABEE I

\begin{tabular}{rrrrrc} 
& $\begin{array}{c}\text { Intradermal } \\
\text { in } \begin{array}{c}\text { Multipuncture } \\
\text { by Grade }\end{array}\end{array}$ & \multicolumn{3}{c}{$\begin{array}{c}\text { Intradermal } \\
\text { in mms. }\end{array}$} & $\begin{array}{c}\text { Multipuncture } \\
\text { by Grade }\end{array}$ \\
I & 9 & I-II & 21 & 5 & I \\
2 & 30 & III & 22 & 15 & I \\
3 & 8 & I-II & 23 & 7 & I \\
4 & 7 & I & 24 & 6 & II \\
5 & 7 & I & 25 & 6 & - \\
6 & 7 & II & 26 & 6 & I \\
7 & 9 & I-II & 27 & 6 & I \\
8 & 8 & II & 28 & 16 & II \\
9 & 6 & I & 29 & 5 & I \\
10 & 7 & I & 30 & 10 & II \\
11 & 6 & II & 31 & 8 & II \\
12 & 7 & I-II & 32 & 6 & I \\
13 & 6 & I & 33 & 6 & I \\
14 & 13 & II & 34 & 6 & I \\
15 & 7 & II & 35 & 6 & I \\
16 & 15 & II & 36 & 7 & I \\
17 & 7 & I & 37 & 14 & III \\
18 & 7 & I & 38 & 9 & I \\
19 & 6 & I & 39 & 8 & II \\
20 & 6 & - & 40 & 5 & -
\end{tabular}

With a little experience, estimating the relative size of the papules is not difficult. It may not appear to be as accurate as measuring with a ruler but that carries a disproportionate margin of error when the infiltration is small. The chief feature of the results is that they broadly corresponded, the larger the infiltration by the intradermal method the more prominent the reaction to the multipuncture tests. The correlation in the majority was sufficient almost to make the three arbitrary grades of positivity equivalent to those of the International Standard.

\section{Simultaneous Lepromin and Tuberculin Testing BY THE Multipuncture Method}

Having demonstrated that lepromin testing by the multipuncture apparatus was feasible and gave results not very different from those by the more orthodox intradermal injection, it was decided to attempt simultaneous lepromin and tuberculin testing by this method. 368 patients in the Buluba and Nyenga Leprosaria were chosen and the lepromin test applied on one forearm and the tuberculin test on the other. The lepromin used was the normal crude 1:20 suspension in isotonic saline. The Tuberculin was PPD (Weybridge) as used in the Heaf Tuberculin Test. The apparatus was the Heaf multipuncture instrument. Separate and new instruments were used for each antigen so that there could be no contamination. 
For purposes of comparison only the late lepromin reaction was used although the early reaction was observed. The results varied with the type and group to which the patients belonged. Where there had been any doubt about the classification, a biopsy had been taken for confirmation.

(a) The Early and Late Lepromin Reactions

\begin{tabular}{llcc} 
& & $\begin{array}{c}\text { Number Positive } \\
\text { at } 48 \text { hours }\end{array}$ & $\begin{array}{c}\text { Number Positive } \\
\text { at } 3 \text { to } 4 \text { weeks }\end{array}$ \\
Lepromatous & $\ldots$ & 17 & 11 \\
Dimorphous & $\ldots$ & 8 & 10 \\
Indeterminate & $\ldots$ & 34 & 53 \\
Tuberculoid & $\ldots$ & 83 & 157 \\
\multicolumn{1}{c}{ Total } & $\ldots$ & $\overline{142}$ & -231
\end{tabular}

(The early lepromin response differs from that due to tuberculin in that it reaches its maximum at 48 hours not 72 ).

An interesting feature was that 15 of the lepromatous patients had a Grade IV response to PPD with blistering and of these 13 had an exaggerated response to the lepromin in which the papules coalesced and there was diffuse infiltration. This did not occur when the lepromin test was done alone. It was regarded as allergic in origin precipitated by the tuberculin. In 8 of these patients the lepromin was still positive four weeks later although very much subsided. $\mathrm{X}$-Ray of the chests showed active tuberculosis in one, a healed focus in five and no change in the others. No reactivation at the site of old lepromin tests was observed.

Sixty three per cent more gave late positive reactions at three to four weeks. A small number of the early positives faded completely which was in line with earlier experience. The fewer positives with the earlier reading and the possibility of allergic interference make the late reading more valuable for estimating the immunological status and particularly the resistance of the individual .

\section{TABLE II}

(b) Comparison of Tuberculin and Late Lepromin Results

\begin{tabular}{|c|c|c|c|c|c|}
\hline & $\begin{array}{c}\text { Tuberculin } \\
\text { Positive }\end{array}$ & $\begin{array}{c}\text { Tuberculin } \\
\text { Negative }\end{array}$ & $\begin{array}{c}\text { Lepromin } \\
\text { Positive }\end{array}$ & $\begin{array}{l}\text { Lepromin } \\
\text { Negative }\end{array}$ & Total \\
\hline Lepromatous & 36 & 23 & 11 & 48 & 59 \\
\hline Dimorphous & 11 & 11 & 10 & 12 & 22 \\
\hline Indeterminate & 50 & 25 & 53 & 22 & 75 \\
\hline Tuberculoid & 132 & 48 & 157 & 23 & 180 \\
\hline Total & 229 & 107 & 231 & 105 & 336 \\
\hline
\end{tabular}




\begin{tabular}{lcccccc} 
& Tuberculin & Tuberculin & Tuberculin & Tuberculin & Total \\
& Positive & Negative & Positive & Negative & \\
& Lepromin & Lepromin & Lepromin & Lepromin & \\
Positive & Negative & Negative & Positive & \\
Lepromatous & 11 & 23 & 25 & 0 & 59 \\
Dimorphous & 7 & 8 & 4 & 3 & 22 \\
Indeterminate & 38 & 10 & 12 & 15 & 75 \\
Tuberculoid & 120 & 11 & 12 & 37 & 180 \\
\multicolumn{1}{c}{ Total } & 176 & 52 & 53 & 55 & 336
\end{tabular}

The proportion of Lepromin positives increased in the order Lepromatous $(20 \%)$ Dimorphous $(45 \%)$ Indeterminate $(70 \%)$ Tuberculoid $(93 \%)$. There was less variation among the Tuberculin positives. It was not possible in this series to correlate with the age groups.

The relationship of one test to the other varied with the type and group. This variation is seen in the increasing proportion that were positive to both as one passed from the lepromatous to the tuberculoid type (Lepromatous $20 \%$, Dimorphous $32 \%$, Indeterminate $50 \%$, Tuberculoid $67 \%$ ) and the decreasing proportion that were negative to both (Lepromatous 40\%, Dimorphous $36 \%$, Indeterminate $13 \%$, Tuberculoid $6 \%$ ). In the same direction, there was a decrease in the proportions that were Tuberculin positive and Lepromin negative (Lepromatous $42 \%$, Dimorphous $18 \%$, Indeterminate $17 \%$, Tuberculoid $7 \%$ ) and an increase in those that were Tuberculin negative Lepromin positive (Lepromatous 0, Dimorphous $14 \%$, Indeterminate $20 \%$, Tuberculoid $20 \%$ ). It is recognised that the dimorphous group was much smaller than the others and percentages are only used to make comparison easier.

In 228 patients, two thirds of the series, the tests coincided, both being positive or both negative. The remaining third was divided equally between those that were positive to one and negative to the other, or negative to one and positive to the other. The agreement was at least as great as could have been expected, in some groups it was greater. The measure of agreement was generally less than that reported for groups of healthy individuals.

The parallelism in two thirds again suggests a relationship between the two diseases. The important groups, however, are the one sixth (52) who were negative to both tests and the other sixth (53) who were tuberculin positive but lepromin negative. These two groups outline the problem of detecting those whose resistance is weak. Among the general population, the individual who is negative both to tuberculin and lepromin can be vaccinated without risk with the assurance that the majority will convert to lepromin positive. A few 
will not, however, despite tuberculin conversion. On the other hand, the individuals who are positive to tuberculin and negative to lepromin have failed to become lepromin positive in the presence of a natural infection with tubercle bacilli. It is hardly to be expected, therefore, that they will convert with BCG. The attempt to vaccinate them will produce a Koch reaction. There would also be the danger of breaking down a quiescent focus, a danger which may be more theoretical than real. Either occurrence would not enhance the popularity of the procedure. This group might be dealt with by repeated oral vaccination or by an antigen of killed Stefansky bacilli combined with a smaller dose of BCG on the lines of that suggested by Hanks.

In any case, it is advisable to discover those at risk at a much earlier age before there has been a primary infection with tubercle. For this, tests are required which are simple to apply and without inconvenience to the individuals who, in most cases, will be the uninfected children in the families of patients. The multipuncture tests appear to fulfil these criteria. Both can be done together or the Heaf tuberculin test can be done when the result of the lepromin test is known. For this purpose grading into degrees of positivity is not necessary. All that is required is to pick out the individuals who are likely to benefit by vaccination and who can be vaccinated without risk.

\section{The Use of Dilute and Depot Lepromins}

Using the multipuncture method, one batch of lepromin diluted to $1: 100$ to which $10 \%$ of glycerine had been added gave more pronounced reactions than the ordinary 1:20 saline suspension. There was no obvious explanation but there are at least three possibilities:-

(a) the added glycerine released more bacilli from the tissue debris and bacillary clumps;

(b) the glycerine had a vasoconstrictor effect and thus kept the antigen longer in contact with the tissues of the patient;

(c) the glycerine in some other way had a depot effect.

It was also found that when intradermal and percutaneous tests were done side by side, the reaction to the former frequently suppressed any reaction to the latter, as though in some patients all available antibodies were attracted to and used up by the larger dose. This was much less obvious when the tests were done in different parts of the body although there was some evidence that the suppression phenomenon was not entirely local in origin.

Twenty-seven patients were chosen at random and tested percutaneously on the back with normal 1:20 lepromin and on the arm with a dilution of $1: 100$ in isotonic saline. 17 were positive to the $1: 20$ antigen, 9 were also positive to the $1: 100$ dilution. 10 were negative to both. Three weeks later the same patients were tested on the back 


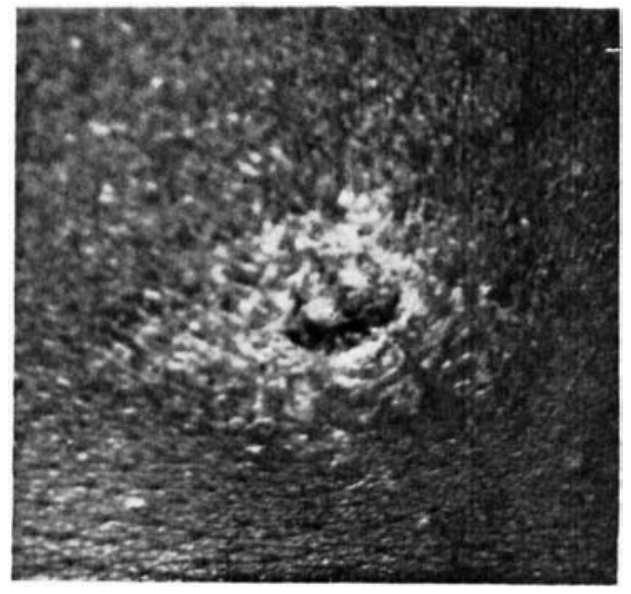

The late Mitsuda with ulceration

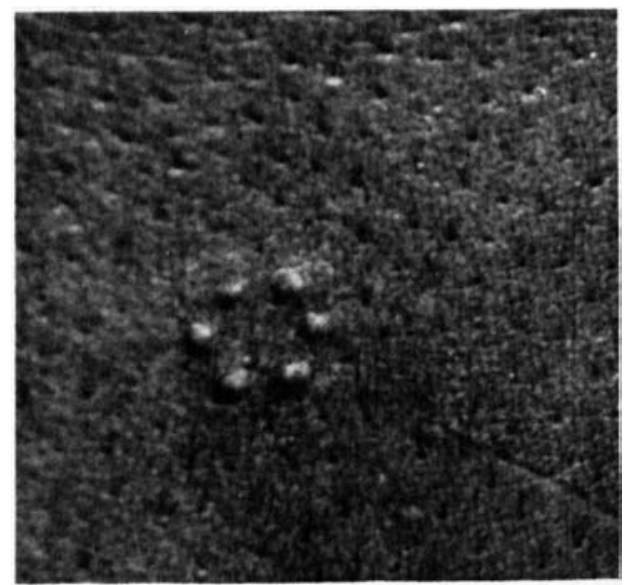

Multipuncture Response Grade II

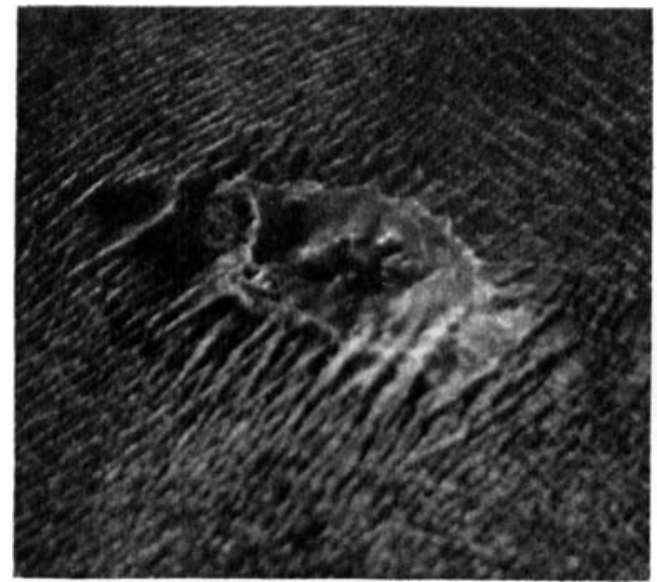

The late Mitsuda with ulceration

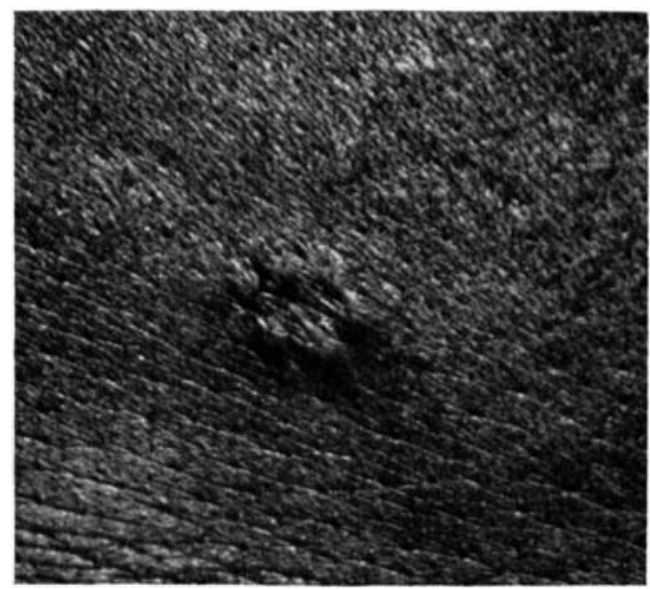

Multipuncture Response Grade III Papules coalescing and pinpoint elevation 


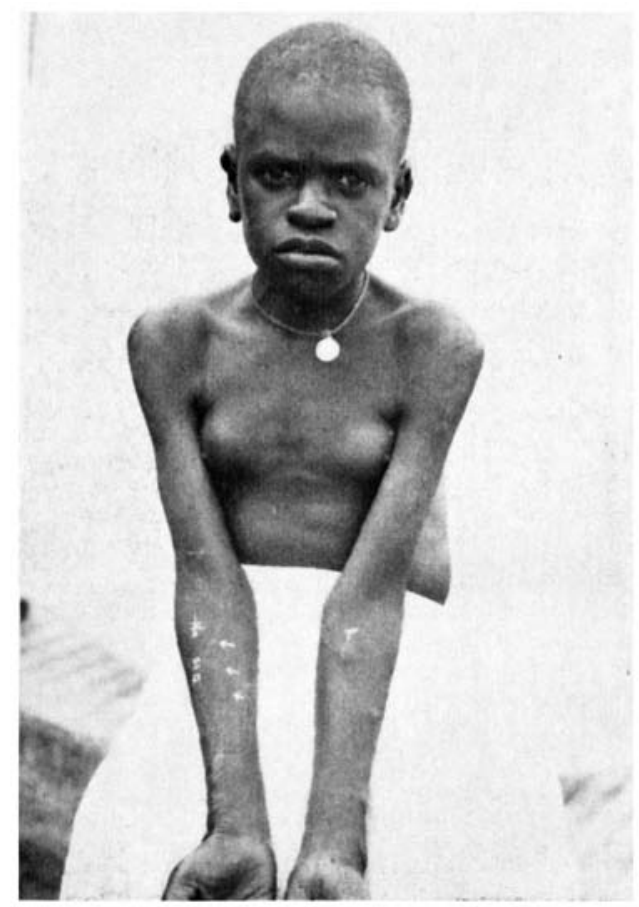

Boy who had a Group IV respense to the Heaf tuberculin at 72 hours, and an exaggeratedresponse to the multipuncture lepromin in clilutions of 1:20, 1:100 and 1:300, at the same time. He was normally lepromin negative ar 4 weeks and as can be seen he was obviously lepromatous with bilateral gynaccomastia. His $x$-ray showed active mberculosis. The exaggerated lepromin response was allergic, due to coincident unberculosis and the P.P.D. 


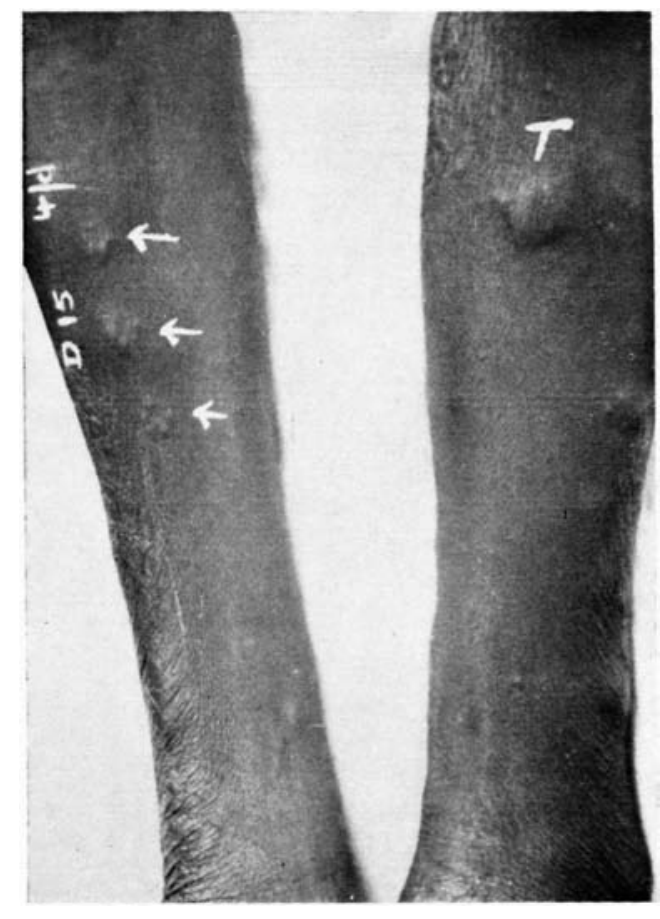

Boy who had a Group IV response to the Heaf tuberculin at 72 hours, and an exaggerated response to the multipuncture lepromin in dilutions of $1: 20$, $1: 100$, and 1:300, at the same time. He was $1: 100$, and $1: 300$, at the same time. He was
normally lepromin negative at 4 weeks and as can be seen he was obviously lepromatous with bilateral gynaecomastia. His $x$-ray showed active tuberculosis. The exaggerated lepromin response was allergic, due to coincident tuberculosis and the P.P.D.

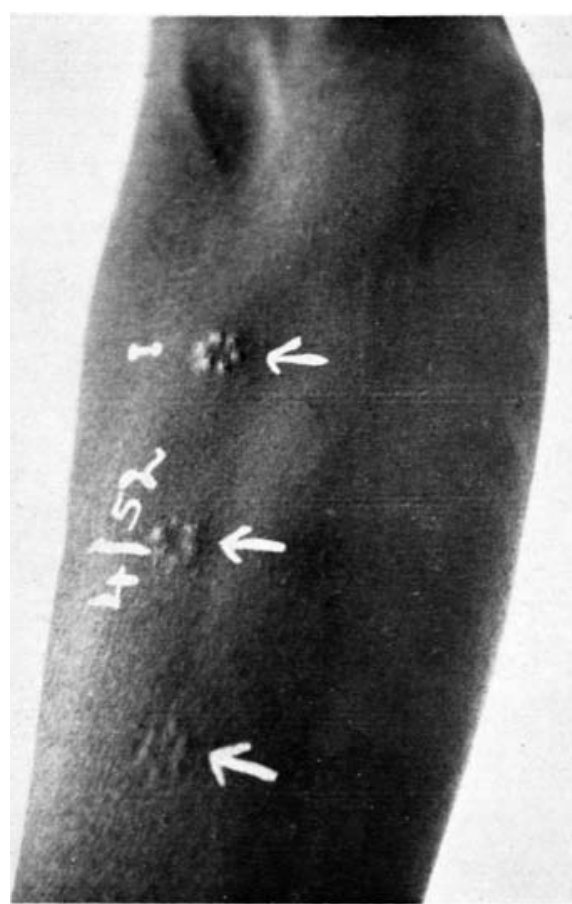

Pilot Tests. Grades I, II, III with different dilutions at 4 weeks. Pin point elevation in 1:20 
with glycerinated lepromin in dilutions of $1: 20$ and $1: 100$. The same 17 were positive to the stronger antigen but the number positive to the 1:100 dilution had increased to 13. The lepromin used in all the tests was from the same batch.

More positives were obtained when glycerine was included in the diluted antigen than when it was left out, although on this occasion the response to $1: 100$ glycerinated lepromin was less pronounced than that to the stronger preparations.

In this work the glycerine had been added some weeks previously when the lepromin was made; in the earlier series it was added just before applying the test. Without being able to say precisely what the role of the glycerine is, it would appear that if a dilute lepromin is to be used by the percutaneous route $10 \%$ of glycerine should be added at some stage.

It was now decided to try to depot lepromin made similarly to the depot tuberculin described by James and Pepys (1956) on the grounds that if the antigen could be kept in close contact with the epidermal tissues for a longer period, an enhanced response might be obtained with less concentrated preparations. It was also thought that if the absorption of the antigen could be delayed, only one test would be necessary in a mass preventive campaign, because it would first pick out the poor responder and after BCG vaccination, it would indicate whether there was conversion to positive or an improvement in response.

The depot medium consisted of 8 parts of light liquid paraffin B.P. and 1 part of anhydrous lanoline B.P. to $4 \mathrm{ml}$. of which $1 \mathrm{ml}$. of isotonic saline was added. Control multipuncture injections were made with this emulsion in 20 patients of various types, but at the end of six weeks, there had been no trace of any reaction whatever. A 1:20 depot lepromin was then made by grinding $0.25 \mathrm{gm}$. of autoclaved lepromatous tissue in $5 \mathrm{ml}$. of the saline constituted depot medium. Further dilutions were made of $1: 100$ and $1: 500$.

Thirty-nine patients, 36 of whom were positive and 3 negative by the intradermal and percutaneous route and who had also been tested percutaneously with a 1:100 saline diluted lepromin were now injected with the three depot lepromins using the multipuncture apparatus.

The agreement in the responses of 37 of the 39 patients to the saline and depot lepromins in concentrations of 1:20 was complete, 34 were positive to both and 3 negative. The lack of correspondence in the other two-positive originally but negative now to the depot lepromins-was most probably the result of the suppression phenomenon as the tests were made fairly close together and the earlier intradermal and percutaneous 1:20 saline reactions were at their height.

Of the 34 who were positive to the $1: 20$ depot lepromin, 26 were 
positive to $1: 100$ and 19 to $1: 500$. The results are summarized below.

TABLE IIla

Saline Lepromins

Glycerinated Lepromins

$1: 20 \quad 1: 100 \quad 1: 20 \quad 1: 100$

No. of Positive

Reactors

17

9

17

\section{TABLE IIIb}

\begin{tabular}{lccc} 
& \multicolumn{3}{c}{ Depot Lepromins } \\
& $1: 20$ & $1: 100$ & $1: 500$ \\
$\begin{array}{l}\text { No. of Positive } \\
\text { Reactors }\end{array}$ & 34 & 26 & 19
\end{tabular}

(The 17 patients in both parts of IIla are the same and are not included in the 34 in Table IIIb.)

It is not possible at this stage to say whether the response to percutaneous depot lepromin will persist longer than that to ordinary saline lepromin. In many cases, however, the 1:100 depot reaction was more definite than that to $1: 100$ saline, and the response to $1: 500$ depot lepromin in the second series was certainly as good as that to $1: 100$ saline lepromin in the first.

Depot lepromin has thus certain advantages. It is also less difficult to prepare. It spreads more easily on the skin and is, therefore, more economical. Using a tuberculin syringe and needle as a dropper and applying the lepromin to the skin direct, 250 tests were obtained from $1 \mathrm{ml}$. of the $1: 20$ preparation, 25 times the number when the intradermal route is used.

It is probable that a depot lepromin in a concentration of about 1:50 will give the same results as 1:20 and such a strength would be less viscous and somewhat easier to use. On the other hand, if it is desired to employ $\mathrm{BCG}$ in a mass campaign for the prevention of leprosy the use of a 1:100 depot test preparation has much in its favour. It is necessary to protect not only the negative responders, but those who react weakly and a 1:100 strength will pick out those who fall into either group. The disadvantage of a stronger antigen is that its scope is not wide enough. It will only identify those who do not react at all. A 1:100 depot lepromin is also even more economical for $1 \mathrm{ml}$. of 1:20 preparation diluted down will provide sufficient material for over 1,000 tests. The great advantage of this test is that it makes practicable a pilot scheme for using BCG prophylactically against leprosy in countries like Uganda.

The problem of the individual who is negative to lepromin and positive to tuberculin, and the individual who is negative to both lepromin and tuberculin and does not convert with BCG has been already referred to. Nevertheless, if only $50 \%$ of negative lepromin 
reactors can be converted to positive, a big step forward will have been taken. There is evidence that those who do convert are much less likely to contract leprosy and if they do, it will be in its less infectious form. The BCG prophylaxis of leprosy linked with the provision of regular and continuous treatment for all who need it should vastly reduce the number of potential patients and the number from whom the disease can be contracted. Hanks described himself as being in the cruel dilemma of knowing how to protect the poor responder but of not knowing how to discover him. It is believed that this modification of the lepromin test will, to a large extent, provide the answer.

\section{SUMMARY}

1. A Series of investigations is described using various lepromins by the intradermal and multipuncture (percutaneous) routes.

2. When the two methods were used simultaneously, the reaction to the bigger dose appeared to suppress any to the smaller. This was more marked when the tests were placed near together.

3. In some patients $10 \%$ glycerine added to $1: 100$ isotonic saline solution enhanced the response to the percutaneous injection.

4. A 1:100 lepromin in isotonic saline can be used as effectively by the intradermal method as the 1:20 concentration and correlation can be established quite simply.

5. The multipuncture (percutaneous) test with 1:20 saline lepromin correlates completely with the 1:20 intradermal test. The multipuncture (percutaneous) test with the 1:20 depot lepromin correlates completely with the 1:20 isotonic saline lepromin by the same route.

6. The multipuncture (percutaneous) test with 1:100 depot lepromin is adequate to detect all the negative reactors and the majority of those who respond weakly: that is, those who most need protection.

7. This modification of the traditional Mitsuda is advocated for field work and where lepromin is in short supply. It is extremely economical of antigen and can, therefore, be used on a very wide scale. It is simple and quick to apply and read. It gives no inconvenience to the individual in the form of a painful and indolent ulcer and is, therefore, not likely to antagonise public opinion in a preventive campaign. It can be used side by side with the Heaf tuberculin test.

8. It is believed that this test opens the door to pilot schemes for trying BCG in the prevention of leprosy and watching the results in both groups of lepromin negatives, i.e. in those who convert and those who persist.

9. The problem of the individual persistently negative to lepromin and positive to tuberculin is discussed. 


\section{ACKNOWLEDGMENTS}

This work has occupied a period of nearly four years. It was intended originally to present it in a series of papers in which reference could more easily be made to those who had contributed. For simplicity in reading, however, it was decided to compress the papers into one. Parts I and III were carried out in conjunction with Dr. W. M. Blenska of the Buluba and Nyenga Leprosaria and could not have been done without her help. Dr. J. M. Lea and Sister M. M. Stone of the Kumi-Ongino Leprosarium assisted with some of the work in Part I. Dr. J. M. Lea gave access to his patients for the work recorded in Parts II and IV and Sister M. M. Stone carried out the day to day routine with patience and thoroughness. To all these, I wish to express my gratitude. I am grateful also to Dr. G. Murray Short, Medical Officer Tuberculosis (Uganda) for his advice at various stages and to Dr. J. Pepys of the Medical Research Council, Tuberculosis Laboratories, for his interest and for providing the depot medium.

\section{REFERENCES}

Diniz, O., and Neto, H. A. 'Results of the use of diluted Mitsuda antigens' Internat. J. of Leprosy, 22, 2, 1954.

FLoch, $\mathrm{H}$. Discussion sur les résultats obtenus dans la réaction de Mitsuda à l'aide d' antigens dilués. Internat. J. of Leprosy, 22, 2, 1954.

HANKS, J. H. Immunological and Physiological Basis of Immunisation in Tuberculosis and Leprosy. Experimental Tuberculosis with an addendum on Leprosy. p. 382, Ciba Foundation Symposium, London, 1955.

Schujman, S. The use of dilute antigens in Lepromin tests. Internat. J of Leprosy, 23, 3, 1955.

James D. Geraint and Pepys, J. Tuberculin in Aqueous and Oily Solutions, The Lancet, 5th May, 1956, 270, 6923. 